\title{
Aproximación a la Tendencia Secular del Estado Nutricional y Composición Corporal en Escolares de Enseñanza Secundaria, V Región, Chile: 1985-2010
}

\author{
Approximation to the Secular Tendency of the Nutritional State \\ and Body Composition of High School Students, V Region, Chile: 1985-2010
}

"Pablo José Lizana Arce; *Atilio Aldo Almagià Flores; **María Cristina Simpson Lelievre; ***** Octavio Binvignat Gutiérrez; ${ }^{* * * *}$ Daniza Ivanovic Marincovich \& ${ }^{* * * * *}$ Francisco José Berral de la Rosa

LIZANA, A. J. P.; ALMAGIA, F. A. A.; SIMPSON, L. M. C. BINVIGNAT, G. O. IVANOVIC, M. D. \& BERRAL DE LA ROSA, F. J. Aproximación a la tendencia secular del estado nutricional y composición corporal en escolares de enseñanza secundaria, V Región, Chile: 1985-2010. Int. J. Morphol., 29(2):473-478, 2011.

RESUMEN: Durante el último siglo se ha identificado una tendencia secular positiva en el crecimiento físico de la mayor parte de las poblaciones del mundo, sobre todo en niños y adolescentes. La situación en Chile no ha sido distinta reflejándose un aumento en todos los grupos etáreos constituyendo uno de los más serios problemas de salud pública en Chile. Frente a este problema, es que se observa la tendencia del estado nutricional y composición corporal de dos muestras de estudiantes de enseñanza media de las comunas de Valparaíso, Viña del Mar y Concón, V Región, Chile, en los períodos 1984-1985 y 2009-2010. Las cohortes presentan diferencias significativas con un aumento en las variables IMC (Índice de Masa Corporal) y porcentaje de grasa corporal $(\mathrm{p}<0,05)$ en la cohorte 2009-2010. Este aumento tiene mayor predominancia en mujeres.

PALABRAS CLAVE: Tendencia secular; Composición corporal; Obesidad; Escolares.

\section{INTRODUCCIÓN}

Durante el último siglo se ha identificado una tendencia secular positiva en el crecimiento físico de la mayor parte de las poblaciones del mundo (Fleta et al., 2000; ZhenWang \& Cheng-Ye, 2005; Simsek et al., 2005; Chrzanowska et al., 2007; Boddy et al., 2009). Esta tendencia de crecimiento también ha incurrido en el aumento en factores de riesgos en la salud, donde en algunas poblaciones incluso ha llegado a triplicarse la prevalencia de sobrepeso y obesidad (Kautiainen et al., 2009). Este fenómeno se ha reportado independiente de la condición socioeconómica (Kautiainen et al.) o en otras, con dependencia, inclinándose a sujetos de bajos recursos y menor nivel educativo (KleinPlatat et al., 2003). La situación en Latinoamérica y Chile no ha sido tan distinta del resto del mundo, los cambios socioeconómicos y la globalización han generado serios cambios de hábitos alimenticios y físicos, que se asemejan a estilos de vida de países desarrollados, lo que conlleva a una malnutrición por exceso y un aumento en el sedentarismo. Esto se refleja en todos los grupos etarios (Albala et al., 2002; Kain et al., 2003; Barría \&, Amigo, 2006), constituyendo uno de los más serios problemas de salud pública en Chile (MINSAL, 2003).

A pesar de que en varias poblaciones del mundo se ha determinado una positiva tendencia secular en niños y adolescentes, Chile no cuenta con datos comparativos de estado nutricional y composición corporal. En este sentido, en este estudio se aborda cómo se ha desarrollado la tendencia del estado nutricional y composición corporal en estudiantes de secundaria de 15 a 18 años de la V Región, Chile.

\footnotetext{
Laboratorio de Antropología Física y Anatomía Humana; Laboratorio de Técnicas Anatómicas, Instituto de Biología, Facultad de Ciencias, Pontificia Universidad Católica de Valparaíso. Chile.

** Instituto de Estadística, Pontificia Universidad Católica de Valparaíso. Chile.

*** Facultad de Ciencias de la Salud. Universidad Autónoma de Chile.

${ }_{* * * * *}^{*}$ Instituto de Nutrición y Tecnología de los Alimentos, Universidad de Chile. Chile.

****** Departamento de Deporte e Informática. Universidad Pablo de Olavide (UPO). Sevilla, España.
} 


\section{SUJETOS Y MÉTODO}

Se tomaron dos muestras transversales y aleatorias de ambos géneros en los períodos 1984-1985 y 2009-2010. La población comprende estudiantes de $1^{\circ}$ a $4^{\circ}$ de educación secundaria de 15 a 18 años de edad (Tabla I) del sistema escolar municipalizado, particular subvencionado y particular pagado. Los participantes son de las comunas de Valparaíso, Viña del Mar y Concón, V Región, Chile, matriculados en el sistema escolar de las comunas mencionadas. Deben disponer del consentimiento informado del o los apoderados, de ellos y del establecimiento educacional, aprobado por el comité de Ética de la Pontificia Universidad Católica de Valparaíso.

Los sujetos son evaluados por antropometría, para esto se considera en la ficha técnica o proforma, nombre, sexo, fecha de nacimiento, fecha de evaluación, edad en años, meses y días, talla en inspiración máxima, peso con el mínimo de ropa, pliegues: tríceps y pierna medial en puntos descritos y estandarizados por Norton \& Olds (1996). Las mediciones fueron realizadas en el hemicuerpo derecho y en los mismos establecimientos educacionales durante la mañana.

El peso se evaluó mediante una balanza de precisión (SECA ${ }^{\mathrm{TM}}$ ) con sensibilidad de 100 gr. Por su parte la talla se valoró mediante un cartabón de $0,1 \mathrm{~cm}$. Para el cálculo del IMC (Peso $(\mathrm{kg}) /$ Talla $^{2}(\mathrm{~cm})$ ) y el estado nutricional se valoró utilizando el referente CDC/2000 (Unidad de Nutrición del Ministerio de Salud MINSAL, 2004).

Tabla I. Distribución de las cohortes estudiadas por género, edad y sexo.

\begin{tabular}{lccc}
\hline Edad & $\mathbf{1 9 8 4 - 1 9 8 5}$ & $\mathbf{2 0 0 9 - 2 0 1 0}$ & Total \\
\hline Niñas & $\mathrm{n}$ & $\mathrm{n}$ & $\mathrm{n}$ \\
15 & 15 & 19 & 34 \\
16 & 23 & 30 & 53 \\
17 & 22 & 29 & 51 \\
18 & 11 & 8 & 19 \\
Total niñas & 71 & 86 & 157 \\
Niños & $\mathrm{N}$ & $\mathrm{N}$ & $\mathrm{N}$ \\
15 & 17 & 21 & 38 \\
16 & 29 & 27 & 56 \\
17 & 24 & 22 & 46 \\
18 & 16 & 7 & 23 \\
Total niños & 86 & 77 & 163 \\
Total de sujetos & & & 320 \\
evaluados & & & \\
\hline
\end{tabular}

La talla se evaluó en bipedestación, descalzos y con mínimo de ropa. El tallímetro transportable fue utilizado en una pared rígida, con una precisión de $0,1 \mathrm{~cm}$.

Para la determinación de la composición corporal, que en el caso de este estudio nuestro principal foco de atención fue registrar el porcentaje de grasa corporal (\%GC), se utilizó la ecuación de Slaughter et al. (1988).

El análisis estadístico se realizó con el programa SPSS versión 15.00 para Windows XP 2002. Se consideró significativo un $\mathrm{p}<0,05$.

\section{RESULTADOS}

Los efectos de los factores estudiados sobre las variables en estudio en la Tabla II.

En la Tabla III se observa la comparación de la distribución de \%GC, según la clasificación de Lohman 1987, en mujeres y en hombres en las cohortes 1985-1986/20092010. Tanto para mujeres como para hombres se rechaza la hipótesis de igualdad de distribuciones de \% GC (mujeres $\mathrm{p}=1,13 \times 10^{-16}$ y para hombres $\mathrm{p}=3,52 \times 10^{-7}$ ).

Los resultados del IMC de los sujetos estudiados, clasificados en P10 (déficit), entre P10 y P85 (normal) y mayores a P85 (sobrepeso y obesidad) comparadas, para los mismos grupos, con las frecuencias esperadas según la tabla de valores CDC/NCHS2000 (MINSAL, 2004). La comparación de la variable IMC tanto para mujeres (A) como para hombres (B) en el período 1984-1985 no muestra significancia estadística. Sin embargo, en la cohorte 2009-2010 ambos géneros muestran significancia estadística (mujeres: $\mathrm{p}=1,295 \times 10^{-10}$; hombres $\mathrm{p}=0,0148$ ) (Fig. 1).

Tabla II. Efecto de los factores estudiados sobre las variables en estudio.

\begin{tabular}{lcccc}
\hline \multirow{2}{*}{ Factor } & \multicolumn{4}{c}{ Variables } \\
\cline { 2 - 5 } & Peso & Talla & IMC & \% GC \\
\cline { 2 - 5 } & $p$ & $p$ & $p$ & $p$ \\
Período & $0,000^{* *}$ & $0,916 \mathrm{NS}$ & $0,000^{* *}$ & $0,000^{* *}$ \\
Sexo & $0,000^{* *}$ & $0,000^{* *}$ & $0,018^{*}$ & $0,000^{* *}$ \\
Edad & $0,016^{*}$ & $0,182 \mathrm{NS}$ & $0,148 \mathrm{NS}$ & $0,121 \mathrm{NS}$ \\
\hline
\end{tabular}

(*) $\mathrm{p}<0,05,(* *) \mathrm{p}<0,01$, (NS) no significativo. 
A

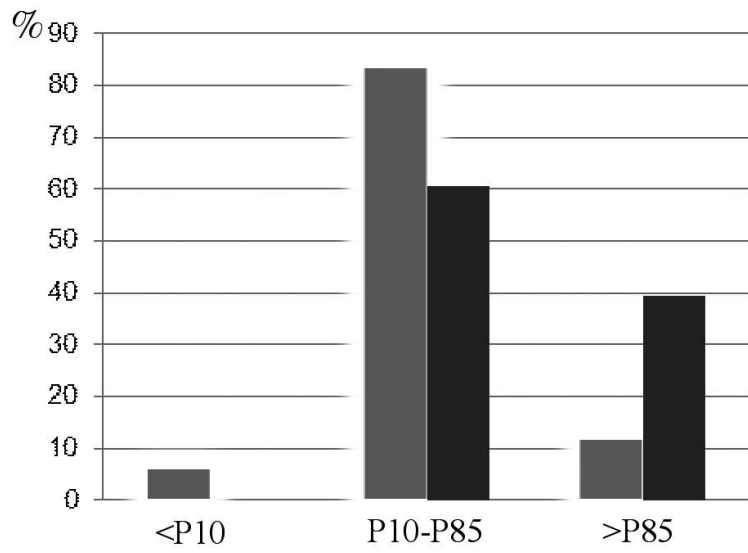

B

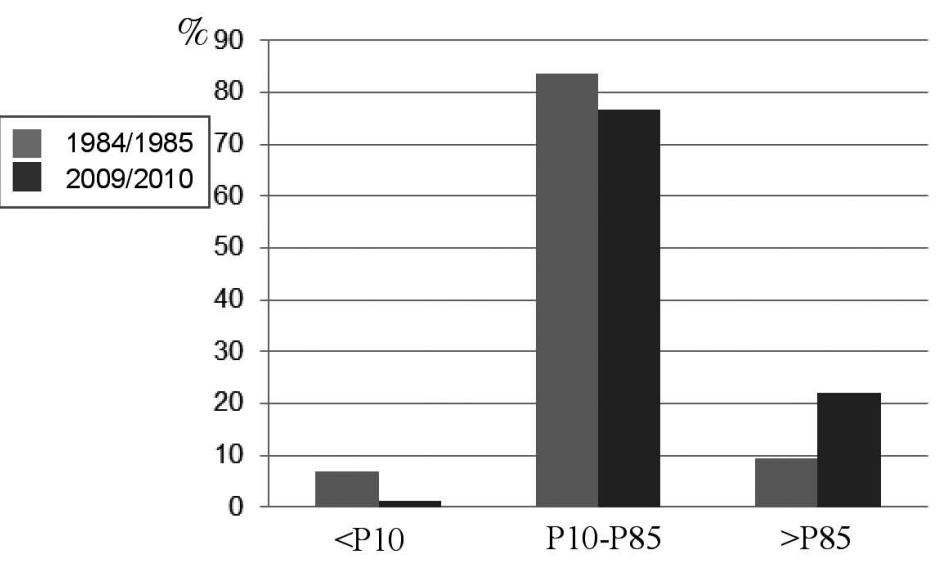

Fig. 1. IMC de los sujetos estudiados, clasificados en P10 (déficit), entre P10 y P85 (normal) y mayores a P85 (sobrepeso y obesidad) comparadas, para los mismos grupos, con las frecuencias esperadas según la tabla de valores CDC/NCHS2000. La comparación de la variable IMC tanto para mujeres (A) como para hombres (B) en el período 1984-1985 no muestra significancia estadística. Sin embargo, en la cohorte 2009-2010 ambos géneros muestran significancia estadística (mujeres: $p=1,295 ¥ 10-10$; hombres $p=0,0148$ ).

Tabla III. Comparación de la distribución de \%GC, según la clasificación de Lohman (1987), en mujeres y en hombres en las cohortes 1985-1986/2009-2010. Tanto para mujeres como para hombres se rechaza la hipótesis de igualdad de distribuciones de \% GC (mujeres $\mathrm{p}=1,13 \times 10^{-16}$ y para hombres $\left.\mathrm{p}=3,52 \times 10^{-7}\right)$.

\begin{tabular}{lcccc}
\hline Distribución & \multicolumn{2}{c}{ Mujeres } & \multicolumn{2}{c}{ Hombres } \\
\cline { 2 - 5 } & $\mathbf{1 9 8 5}$ & $\mathbf{2 0 1 0}$ & $\mathbf{1 9 8 5}$ & $\mathbf{2 0 1 0}$ \\
\hline Muy bajo & 16 & 0 & 9 & 0 \\
Bajo & 7 & 0 & 41 & 18 \\
Óptimo & 46 & 27 & 34 & 39 \\
Moderadamente alto & 2 & 23 & 1 & 8 \\
Alto & 0 & 23 & 1 & 5 \\
Muy alto & 0 & 13 & 0 & 7 \\
\hline
\end{tabular}

\section{DISCUSIÓN}

El objetivo de esta investigación fue evidenciar la tendencia del estado nutricional entre los años 1984-1985/ 2009-2010 a través del IMC y composición corporal, período en que han ocurrido cambios importantes en los estilos de vida de la población Chilena (Albala et al.; Kain et al., 2005; Rodríguez \& Pizarro, 2006; Barría \& Amigo). Este período denominado de post-transición se ha caracterizado por un aumento de las enfermedades crónicas no transmisibles (ECNT) como afecciones cardiovasculares, diabetes tipo II, cáncer y obesidad (Albala et al.). Estos cambios en los estilos de vida también pueden producir cambios en una pubertad temprana (Burrows et al., 2010).

La comparación entre las cohortes para todas las va- riables muestran diferencias significativas aspectos que ya pudieron ser visualizados por diversos autores (Fleta et al.; Zhen-Wang \& Cheng-Ye; Simsek et al.; Chrzanowska et al., 2007; Boddy et al.) excepto la talla que no alcanzó significancia estadística. Si el análisis se enfoca en la variable peso, se evidencia un notable aumento pasando de una media de 59,38 kg en los años (1984-1985) a 65,62 kg en la cohorte (2009-2010) con una diferencia significativa entre los grupos de 6,23 kg. Sin embargo, si se desglosa por sexo, se aprecia que este aumento de peso presenta un dimorfismo sexual. Es así, que los hombres presentan una media de 64,20 kg en el primer período estudiado pasando a $67,45 \mathrm{~kg}$ para el segundo periodo con una diferencia de 3,26 kg Esta diferencia se ve aumentada en las mujeres donde en el primer periodo presentan una media de $54,57 \mathrm{~kg}$ y para el segundo periodo estudiado $63,78 \mathrm{~kg}$, lo que contempla un aumento de 9,21 kg, un incremento de aproximadamente el triple en comparación con los hombres. Este progreso en el peso y dimorfismo sexual también ha sido reportado en otras poblaciones (Carrascosa et al., 2004). Sin embargo, a diferencia de los datos de Carrascosa y colaboradores donde sus valores son positivos para los hombres y poco significativo para las mujeres, en este estudio se registra aumentos significativos en ambos géneros con una mayor diferencia en las mujeres.

En cuanto a la talla se observa que entre las cohortes evaluadas no existen diferencias significativas. Sin embargo, prevalece un notable dimorfismo sexual que se mantiene entre hombres y mujeres ( $\mathrm{p}<0,001)$, siendo estas últimas más bajas que los hombres. Esta diferencia ya ha sido observada por otros estudios (Carrascosa et al., 2006) y el que puede deberse a genes para la talla en los cromosomas sexuales (Valenzuela, 1975) 
LIZANA, A. J. P.; ALMAGIA, F. A. A.; SIMPSON, L. M. C. BINVIGNAT, G. O. IVANOVIC, M. D. \& BERRAL DE LA ROSA, F. J. Aproximación a la tendencia secular del estado nutricional y composición corporal en escolares de enseñanza secundaria, V Región, Chile: 1985-2010. Int. J. Morphol., 29(2):473-478, 2011.

Al realizar la comparación de las cohortes por edad para cada una de las variables no presentan diferencias significativas. Por lo tanto, la variabilidad no está influenciada por la edad por lo que el análisis de comparación se realiza en forma conjunta, al igual que lo observado por Carrascosa et al. (2004).

El presente estudio muestra un aumento significativo del IMC en ambos sexos (Fig. 1), lo que coincide con otros estudios internacionales (Chrzanowska et al.; Boddy et al.) y nacionales (Burrows et al.). En este último observaron que en el periodo 1985 - 2001 la obesidad se había cuadriplicado. Esto es consecuente con estudios ingleses donde en el periodo 1998 - 2006 se evaluaron a niños y a niñas de 9 - 10 años durante 5 años visualizando un aumento del IMC, donde las niñas presentaron mayores aumentos que los niños (Boddy et al.) al igual que los datos de este estudio, pero en edades distintas. Si se compara este estudio con el trabajo de Chrzanowska et al. para las mismas edades estudiadas, pero en años diferentes (1971 - 1983 y 2000) ellos no encuentran diferencias significativas en las edades de 14 a 18 años en la población polaca (ciudad de Cracovia). En relación a este fenómeno los autores aluden a una alta preocupación por la estética, debido a una transición de libre mercado en ese país.

Si se toma como referente el CDC/2000 (Unidad de Nutrición del Ministerio de Salud MINSAL) para analizar el IMC entre hombres y mujeres se observa que para los años 1984-1985 no existen diferencias significativas. Sin embargo, para los años 2009-2010 si existen diferencias significativas con un claro dimorfismo sexual (mujeres $\mathrm{p}=$ $1,295 \times 10^{-10}$; hombres $\mathrm{p}=0,0148$; Fig. 1 ).

Para el \%GC en los periodos estudiados existen diferencias significativas entre mujeres y entre hombres para cada cohorte estudiada $\left(\mathrm{p}=1,13 \times 10^{-16} ; \mathrm{p}=3,52 \times 10^{-7}\right.$ respectivamente). Existiendo un predominio de porcentaje de grasa corporal en las mujeres. Es así, que, si agrupamos los rangos de riesgo (moderadamente alto, alto y muy alto) pasamos de un $2,82 \%$ a un $68,6 \%$ en las mujeres y de un $2,33 \%$ a un $25,97 \%$ en los hombres. Esto indica un dimorfismo sexual entre hombres y mujeres, que ya había sido identificado por el grupo de Almagià et al. (1977) para la población de Valparaíso, pero con las fórmulas de Durning $\&$ Womersley (1974) que son para adultos por lo que no se permite hacer comparaciones. En otras poblaciones también se ha visualizado este patrón diferenciado entre hombres y mujeres (Berral et al., 2001; Moreno et al., 2006) utilizando la misma fórmula de Slaughter para el cálculo de $\% \mathrm{GC}$.

Debido a que este estudio es exploratorio y preliminar presenta limitaciones en su tamaño muestral en la distribución del IMC y \%GC. En el primero se fusionó riesgo de sobrepeso con obesidad y en el segundo las categorías muy bajo con bajo y moderadamente alto, alto y muy alto lo que será disgregado con un n mayor. Sin embargo, se aprecian altas prevalencias de sobrepeso y obesidad ( $\mathrm{p}>85$ ). Además de elevados rangos de grasa corporal, sobre todo en mujeres, lo que hace urgente aplicar medidas para tratar este aumento de grasa corporal, como también instaurar el protocolo de \%GC como diagnóstico más exacto del estado nutricional del niño y adolescente.

\section{AGRADECIMIENTOS}

A la Dirección de Investigación, Vice-rectoría de Investigación y Estudios Avanzados, Instituto de Biología de la Facultad de Ciencias, Pontificia Universidad Católica de Valparaíso. Proyecto de investigación financiado por FONIS - Fondo Nacional de Investigación y Desarrollo en Salud CONICYT (Proyecto FONIS SA10I20005). Por el apoyo y financiamiento.

LIZANA, A. J. P.; ALMAGIA, F. A. A.; SIMPSON, L. M. C. BINVIGNAT, G. O. IVANOVIC, M. D. \& BERRAL DE LA ROSA, F. J. Approximation to the secular tendency of the nutritional state and body composition of high school students, V Región, Chile: 19852010. Int. J. Morphol., 29(2):473-478, 2011.

SUMMARY: Throughout the last century a positive secular tendency has been identified in the physical growth in most part of the world population, specifically in children and teenagers. The situation in Chile has not differed from the universal reality, showing a rise in all age groups constituting one of the most serious problems of Chile's public health system. In light of that issue, the tendency of the nutritional state and body composition of two groups of high school students from Valparaiso, Viña del Mar and Concón, V región, Chile, between the periods 1984/1985 and 2009/2010 have been studied. The cohorts present significant differences with an increase in the variables of BMI (Body Mass Index) and body fat percentage $(\mathrm{p}<0,05)$ in the 2009/2010 cohort. This rise is more predominant in women.

KEY WORDS: Secular tendency; Body composition; Obesity; Students. 
LIZANA, A. J. P.; ALMAGIA, F. A. A.; SIMPSON, L. M. C. BINVIGNAT, G. O. IVANOVIC, M. D. \& BERRAL DE LA ROSA, F. J. Aproximación a la tendencia secular del estado nutricional y composición corporal en escolares de enseñanza secundaria, V Región, Chile: 1985-2010. Int. J. Morphol., 29(2):473-478, 2011.

\section{REFERENCIAS BIBLIOGRÁFICAS}

Albala, C.; Vio, F.; Kain, J. \& Uauy, R. Nutrition transition in Chile: determinants and consequences. Public. Health Nutr., 5(1A):123-8, 2002.

Almagià, A.; Gurovich, A.; Ivanovic, D. M.; Toro, T. \& Binvignat, O. Estudio y análisis morfológico etario del dimorfismo sexual a través de la composición corporal. Rev. Chil. Anat., 15(2):141-9, 1997.

Barría, R. M. \& Amigo, H. Nutrition transition: a review of Latin American profile. Arch. Latinoam. Nutr., 56(1):3-11, 2006.

Berral, F.; Gómez, J.; Viana, B.; Berral, C. \& Carpintero, P. Estudio de la composición corporal en escolares de 10 a 14 años. Rev. Bras. Cineantropom. Desempenho Hum., 3(1):20-33, 2001.

Burrows, R.; Ceballos, X.; Burgueño, M. \& Muzzo, S. Tendencia del desarrollo puberal en escolares de la Región Metropolitana de Chile: Menor edad de presentación, mayor duración y dimorfismo sexual en la estatura. Rev. Méd. Chil., 138(1):61-7, 2010.

Boddy, L. M.; Hackett, A. F. \& Stratton, G. Changes in BMI and prevalence of obesity and overweight in children in Liverpool, 1998-2006. Perspect. Public. Health., 129(3):127-31, 2009.

Carrascosa, A. Obesidad durante la infancia y adolescencia. Una pandemia que demanda nuestra atención. Med. Clin. (Barc), 126(18):693-4, 2006.

Carrascosa, A.; Yeste, D.; Copil, A. \& Gussinyé, M. Aceleración secular del crecimiento. Valores de peso, talla e índice de masa corporal en niños, adolescentes y adultos jóvenes de la población de Barcelona. Med. Clin. (Barc), 123:445-51, 2004.

Chrzanowska, M.; Koziel, S. \& Ulijaszek, S. J. Changes in BMI and the prevalence of overweight and obesity in children and adolescents in Cracow, Poland, 19712000. Econ. Hum. Biol., 5(3):370-8, 2007.

Durnin, J. V. \& Womersley, J. Body fat assessed from total body density and its estimation from skinfold thickness: measurements on 481 men and women aged from 16 to 72 years. Br. J. Nutr., 32(1):77-97, 1974.

Fleta, J.; Rodríguez, G.; Mur, L.; Moreno, L. \& Bueno,
M. Tendencia secular del tejido adiposo corporal en niños prepúberes. An. Esp. Pediatr., 52(2):116-22, 2000 .

Kain, J.; Uauy, R.; Lera, L.; Taibo, M.; Espejo, F. \& Albala, C. Evolución del estado nutricional de escolares chilenos de 6 años (1987-2003). Rev. Med. Chil., 133(9):1013-20, 2005.

Kain, J.; Vio, F. \& Albala, C. Obesity trends and determinant factors in Latin America. Cad. Saúde Pública., 19(Supl 1):S77-S86, 2003.

Kautiainen, S.; Koivisto, A. M.; Koivusilta, L.; Lintonen, T.; Virtanen, S. M. \& Rimpelä, A. Sociodemographic factors and a secular trend of adolescent overweight in Finland. Int. J. Pediatr. Obes., 4(4):360-70, 2009.

Klein-Platat, C.; Wagner, A.; Haan, M. C.; Arveiler, D.; Schlienger, J. L. \& Simon, C. Prevalence and sociodemographic determinants of overweight in young French adolescents. Diabetes Metab. Res. Rev., 19(2):153-8, 2003.

Lohman, T. G. The use of skinfold to estimate body fatness on children and youth. J. Phys. Educ. Recreat. Dance,; 58(9):98-102, 1987.

Moreno, L. A.; Mesana, M. I.; González-Gross, M.; Gil, C. M.; Fleta, J.; Wärnberg, J.; Ruiz, J. R.; Sarría, A.; Marcos, A.; Bueno, M. \& AVENA Study Group. Anthropometric body fat composition reference values in Spanish adolescents. The AVENA Study. Eur. J. Clin. Nutr., 60(2):191-6, 2006.

MINSAL, Ministerio de Salud de Chile. I Encuesta de Salud. Santiago: MINSAL. 2003. http://epi.minsal.cl/Epi/ Html/Invest/ENS/InformeFinalENS.pdf

Norton, K. \& Olds, T. Antropometrica. Marrickville, Ed. Southwood Press, 1996.

Rodríguez, L. \& Pizarro, T. Situación nutricional del escolar y adolescente en Chile. Rev. Chil. Pediatr., 77(1):70-80, 2006.

Simsek, F.; Ulukol, B. \& Gulnar, S. B. The secular trends in height and weight of Turkish school children during 1993-2003. Child Care Health Dev., 31(4):441-7, 2005. 
LIZANA, A. J. P.; ALMAGIA, F. A. A.; SIMPSON, L. M. C. BINVIGNAT, G. O. IVANOVIC, M. D. \& BERRAL DE LA ROSA, F. J. Aproximación a la tendencia secular del estado nutricional y composición corporal en escolares de enseñanza secundaria, V Región, Chile: 1985-2010. Int. J. Morphol., 29(2):473-478, 2011.

Slaughter, M. H.; Lohman, T. G.; Boileau, R. A.; Horswill, R. J.; Stillman, M. D.; Van Loan, M. D. \& Bemben, D. A. Skinfold Equations for Estimation of Body Fatness in children and Youth. Hum. Biol., 60(5):709-23, 1998.

Unidad de Nutrición del Ministerio de Salud MINSAL. Consejo Asesor en Nutrición. Norma Técnica de Evaluación Nutricional del Niño de 6 a 18 años 2003. Rev. Chil. Nutr., 31:128-38, 2004.

Valenzuela, C. Y. Dimorfismo sexual pondoestatural en una población chilena ¿Evidencias de genes para la estatura en los cromosomas sexuales? Rev. Med. Chile, 103:3225,1975 .

Zhen-Wang, B. \& Cheng-Ye, J. Secular growth changes in body height and weight in children and adolescents in Shandong, China between 1939 and 2000. Ann. Hum. Biol., 32(5):650-65, 2005.
Dirección para Correspondencia:

Prof. Pablo Lizana Arce. M.Sc.

Laboratorio de Antropología Física y Anatomía Humana Laboratorio de Técnicas Anatómicas

Pontificia Universidad Católica de Valparaíso

Av. Universidad 330

Curauma, Placilla

Valparaíso

CHILE

Email: pablo.lizana@ucv.cl

Recibido : 28-02-2011

Aceptado: 17-03-2011 